\title{
Reconstruction from Limited Projection Data Using Fuzzy Sets
}

\author{
by \\ M. Reha Civanlar \\ and \\ H.J. Trussell
}
Center for Communications and Signal Processing
Department of Electrical and Computer Engineering North Carolina State University

\author{
January 1986 \\ CCSP-TR-86/3
}




\section{Abstract}

Tomographic reconstruction using limited projection data requires the incorporation of all available a priori information in the reconstruction process. The difficulty in using much of the available information, including the projection data, is the lack of a mathematical model for inaccurate or vague data. The use of the fuzzy set theory gives the flexibility to model and incorporate such information in the reconstruction process.

\section{Introduction}

In several cases the available projection data may not be sufficient for an acceptable tomographic reconstruction. This can be because of various constraints including: time constraints as in the case of "stop action" imaging of the heart; physical constraints such as large bones limiting the view; and constraints on the accuracy of measurements because of noise contamination, miscalibration of the detectors or beam hardening. To obtain acceptable reconstructions using a small number of projections is also important in reducing the overall $\mathrm{X}$-ray exposure time of the patient. Clearly, in order to obtain an acceptable reconstruction, the missing information in the limited projection data must be supplied from some other source. This necessitates the use of a reconstruction algorithm which incorporates all available a priori information about the reconstructed image and the projection process.

Recently, in many papers, ${ }^{1,2,3}$ mostly iterative methods are suggested for the limited angle reconstruction problem. These methods can incorporate much of the exact a priori information. However, in practical applications, a great deal of the a priori information is imprecise. Examples include: the range of expected CT numbers (since the composition of human body is known, there are possible and impossible CT numbers that may result from a reconstruction); the shapes of various organs; and the limits on the accuracy of the detectors caused by noise contamination. 
Apart from the a priori knowledge about the scanned object, an uncertainity is introduced because of the assumptions made about the image and the projection process in order to obtain a mathematically tractable reconstruction algorithm. The most popular reconstruction algorithm, the filtered backprojection, assumes a bandlimited image while, none of the practical images are bandlimited because of the edges that they contain. In computing the projections of a reconstructed image, the integrals of a nondifferentiable function must be estimated from its samples. This is not possible unless some assumptions are made about the scanned object and most of these assumptions are not realistic. Solely because of this reason, a fundamental question, whether a reconstructed image corresponds to the recorded projection data, cannot be answered exactly.

Fuzzy set theory is a tool for describing and manipulating imprecision and it is possible to formulate the tomographic reconstruction problem using fuzzy sets. In this formulation, each piece of available information, including the limited projection data, is used to define a fuzzy set of images. The actual image is known to be a member of each of these sets with a high membership value, and thus, it must be a member of the intersection of all of these fuzzy sets. This suggests defining the solution as the member of the intersection set with the highest membership level. The computation of such a solution can be done using the projections onto convex sets (POCS) and other optimization techniques.

\section{A review of the fuzzy set theory}

The characteristic function $\mu_{A}: X \rightarrow\{0,1\}$ of an ordinary (non fuzzy) subset $A$ of a universe $X$ is defined as:

$$
\mu_{A}(x)=\left\{\begin{array}{lll}
1 & \text { if } & x \in A \\
0 & \text { if } & x \in A
\end{array}\right.
$$

where $\{0,1\}$ is called a valuation set. If the valuation set is the interval $[0,1]$ of real 
numbers then $\mathrm{A}$ is called a "fuzzy set" $4 \mu_{A}(x)$ is called the "grade of membership" of $x$ in $A$. Qualitatively, the grade of membership describes the strength of our belief that $x$ is a member of $A$. If $\mu_{A}(x)=1$, it is certain that $x$ is in $A$; and if $\mu_{A}(x)=0$, it is certain that $x$ is not in $A$. Thus, the fuzzy set $A$ can be considered as a subset of $X$ with vague boundaries.

Two fuzzy sets $\mathrm{A}$ and $\mathrm{B}$ are equal iff $\mu_{A}(x)=\mu_{B}(x)$, and $A$ is a subset of $B$ iff $\mu_{A}(x)<\mu_{B}(x), V x \in X$. The "height" of a fuzzy set $A$ is defined as:

$$
\operatorname{hgt}(A)=\sup \mu_{A}(x)
$$

and a fuzzy set is called "normalized" if its height is 1 . The usual definitions for the ordinary set operations are extended to fuzzy sets as follows:

$$
\begin{gathered}
\mu_{A \cup B}(x)=\max \left(\mu_{A}(x), \mu_{B}(x)\right) \\
\mu_{A \cap B}(x)=\min \left(\mu_{A}(x), \mu_{B}(x)\right) \\
\mu_{A}(x)=1-\mu_{A}(x)
\end{gathered}
$$

These operations satisfy most of the usual properties of the ordinary union, intersection, and complementation such as commutativity, associativity and there are alternative definitions for these operators which are also consistent.

Since in this application the intersection of several fuzzy sets is used to describe a reconstruction, the intersection operator is of the most importance. Two alternative definitions for this operator are:

$$
\begin{array}{lcc}
\text { product form }: & \mu_{A B}(x)=\mu_{A}(x) \mu_{B}(x) \\
\text { bold intersection: } & \mu_{A+B}=\max \left(0, \mu_{A}(x)+\mu_{B}(x)-1\right)
\end{array}
$$

The convexity notion of the set theory is generalized to fuzzy sets as follows: a fuzzy set is "convex" iff its $\alpha$-cuts are convex; where the $\alpha$-cut, $A_{\alpha}$, of a fuzzy set $\mathrm{A}$ is defined as:

$$
A_{\alpha}=\left\{x \in X: \mu_{A}(x) \geq \alpha\right\}
$$


$A_{\alpha}$ is an ordinary set.

The size of a fuzzy set is defined by using its entropy and energy measures. Two forms for the energy measure of a fuzzy set are given in eq. 6 and 7 :

$$
\begin{aligned}
& e(A)=\frac{1}{h g t(A)} \int \mu_{A}(x) d x \\
& e(A)=\frac{1}{h g t^{2}(A)} \int \mu_{A}^{2}(x) d x
\end{aligned}
$$

\section{Fuzzy sets for image reconstruction}

\section{Fuzzy sets defined by the projection data}

The most important information about a reconstruction is obviously the recorded projection data. Irrespective of the generation of the tomography machine used, each individual projection measurement can be modeled as a line or strip integral of the image field. The problem in relating a reconstruction to a certain set of projections is the unaccessibility of the continuous form of the image. Since a digital computer must be used in the reconstruction process, it is unavoidable to have a digitized picture. A simplistic approach to estimate the projections corresponding to a digitized picture is to assume a constant intensity inside each square pixel and to compute the integral by summing the product of pixel intensities with the ray lengths (or areas) inside those pixels. This is the model used in early forms of the algebraic reconstruction techniques (ART) ${ }^{5}$. However, even if the final result displayed on the monitor is digital, when the projections are estimated from such a model, the computational error will not be negligible.

A solution for this may be using a natural pixel decomposition ${ }^{6}$ where, the pixels are defined to be the ray paths through the image. The disadvantages of this formulation are caused by the large sizes and the intersecting structure of the pixels. Much of the a priori information such as nonnegativity or a complicated region of support cannot be incorporated in the natural pixels model. To relate the displayed picture to the natural pixels 
model may be easier than to relate it to the continuous picture but it is still nontrivial. There have been attempts to use higher order integration techniques to estimate the projections ${ }^{7}$. It is possible to reduce the error to some extent by using better integration techniques, but because of the nondifferentiable nature of the image, the estimates will always be erroneous.

It is possible to define a fuzzy consistency between the computed projections and the recorded data by developing a probabilistic error model. This model is based on the observation that the important part of the error is caused by the intensity jumps. In fig.1 such an error is demonstrated. In this example, a simple rectangular integration is shown, however; the same reasoning can easily be applied to higher order numeric integrals.

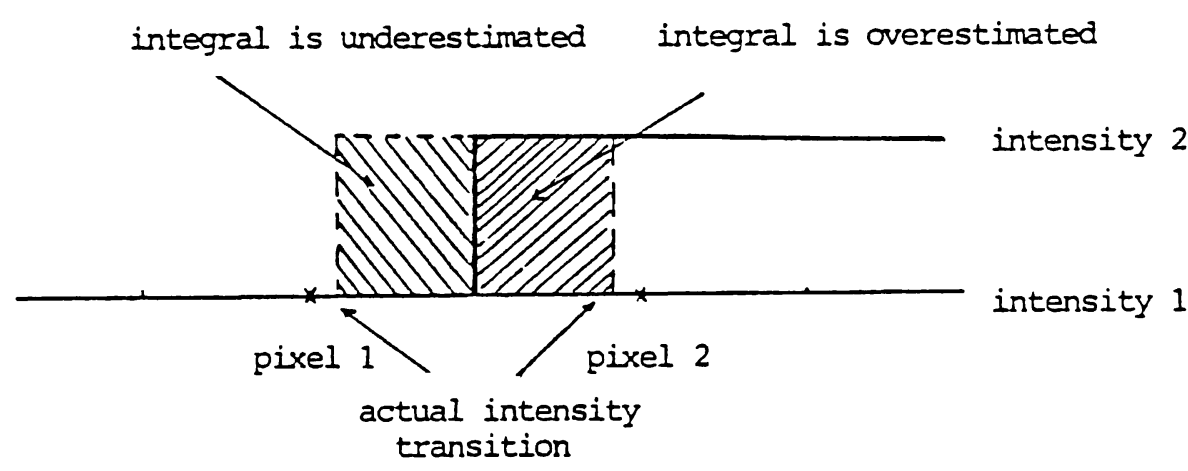

Figure 1 - The integration error caused by an edge.

The maximum value of such an error is proportional to the maximum intensity difference multiplied by the pixel size. However, the actual value is not always equal to the maximum value. Having no other information about the process, it is assumed that, whenever 
a ray encounters an intensity difference, an error which has a Gaussian distribution with a zero mean is made. The standard deviation of the error may be assumed to be one fourth of the largest error because of the fact that the largest error occurs with a very low probability.

The number of intensity differences encountered by each ray can be estimated using an a priori model for the scanned object. To do this, the geometric position of each ray on a picture similar to the picture of the object to be scanned must be considered. In cases where this becomes too involved, a Poisson density for the number of such transitions may be assumed. ${ }^{8}$ In both cases, the final distribution may be approximated by a Gaussian. The fuzzy set of images consistent with a given projection data can then be defined using techniques for generating membership functions from statistical data. ${ }^{9}$ The form of such a membership function is:

$$
\mu(f)=\left\{\begin{array}{cc}
1 & \text { if }\left|a_{i}^{t} f-p_{i}\right|<c_{i} \\
\text { otherwise }
\end{array}\right.
$$

where, $f$ is the reconstructed image considered as a $N^{2}$ vector (assuming a $N x N$ picture with a certain ordering such as a lexicographic order), $a_{i}$ is the vector of weights used to compute the integral of the image over the $i^{\text {th }}$ ray, $p_{i}$ is the $i^{\text {th }}$ projection data, and $\eta_{i}$ and $c_{i}$ are constants determined using the error statistics outlined above.

In addition to the inaccuracy caused by the computation of the projections, because of noise contamination and other non-ideal effects, the recorded projection data are not error free. Such errors can be used to define fuzzy sets of projections which are consistent with recorded projections. For example, if it is known that each projection datum is contaminated by additive Gaussian noise, then the fuzzy set of projections consistent with the recorded data may be described by a membership function of the form: 


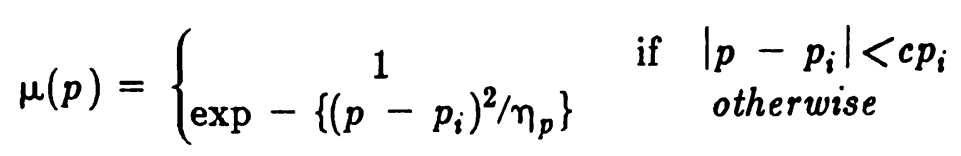

where, $p_{i}$ 's are the recorded data, and $\eta_{p}$ and $c p_{i}$ 's are to be determined from noise statistics.

The final membership function of a reconstructed image can be found by combining the membership functions defined in eq's 8 and 9 using the intersection operator. For the case of ideal error free projections, the membership function of the projections is one only for the recorded projection data and zero everywhere else. For this case, the consistency of the recorded image with the projection data determined only by the integration error.

\section{Fuzzy region of supports}

The region of support, that is the region of the picture where the scanned object is known to lie, is reported to be a very effective constraint. ${ }^{1,2,3}$ One problem with using this information, however; is that because of the possible motion of the patient and measuring and registration problems, it is difficult to define this region exactly. If a larger than actual region is used the effectiveness of this information will be reduced. A fuzzy region of support can be used to obtain a more realistic model for this information. A possible membership function for this set is given by:

$$
\mu(f)=\exp -M f
$$

where, $M$ is a mask defining the degree of belief in having zero intensity at a certain pixel, and $f$ is the image. Larger values in $M$ enforce corresponding pixels to be zero.

\section{Fuzy ranges for CT numbers}

For a certain section of the body, there is an expected range of CT numbers. This information is very important in obtaining a smooth and consistent image. The reason for using a fuzzy set for modeling range of the expected CT numbers is to have a flexibility for handling pixels with out of range values without enlarging the range too much. A 
membership function for this set may be of the form:

$$
\mu(f)=\prod_{i=1}^{N} \sum_{j=1}^{M} \beta_{j} \exp \left(-\alpha_{j}\left([f]_{i}-[f l v]_{j}\right)^{2}\right)
$$

where $f l v$ is a vector containing the $M$ most possible levels, and the $\alpha$; and $\beta$; parameters can be adjusted to give the necessary dispersions around these levels.

\section{Fuzzy frequency domain information}

In many cases the result of a reconstruction has an unacceptable intensity variation. This indicates that there is a priori information about the smoothness of the final picture. This information may be modeled using a fuzzy set with a membership function:

$$
\mu(f)=\exp -W F
$$

where $F$ is a vector containing the magnitudes of the Fourier transform of the reconstruction and $W$ is a vector containing weights for frequency coefficients. The larger the weight the smaller the corresponding frequency component will be.

\section{Computation of the solution}

After defining the fuzzy sets to describe a reconstruction consistent with all available a priori information, the next step is to find an element in the intersection of these sets. In the case of convex fuzzy sets this problem can be solved using the alternating projections onto convex sets (POCS). In this technique, an initial reconstruction is projected onto $\alpha$-level sets of each of the convex fuzzy sets successively. POCS is based on a method for finding common points of closed and convex sets. This method has been applied to the tomographic reconstruction problem ${ }^{\mathbf{7 , 8}}$ and image restoration ${ }^{\mathbf{9}}$ using ordinary sets. The main theorem of the POCS method can be stated as: ${ }^{10,11}$

Let $C_{0}=\cap C_{i}$ be nonempty, where each $C_{i}$ is a closed and convex set in a Hilbert space. Let $P_{i}[$.$] be an operator projecting a vector onto set C_{i}$. Then the iteration given by: 


$$
f^{k}=P_{1} P_{2} \ldots P_{M}\left[f^{k}\right]
$$

converges weakly to a point in $C_{0}$. Clearly, the convergence is strong in finite dimensional spaces. The speed of convergence of this iterative process depends on the sets used. Each individual projection operator is found by solving the following constrained minimization problem:

$$
\min \left\|\underset{f \in C_{k}}{f}-f^{0}\right\|^{2}
$$

where, $f^{0}$ is the image to be projected onto the $k^{\text {th }}$ convex set. The computational complexity of the individual projections also depends on the sets. The computational complexity of the method requires special purpose computing architectures for reasonable computation times. This is not an extraordinary requirement because ordinary scanners also use similar special purpose devices.

If some of the fuzzy sets used in the formulation are not convex, a numeric optimization algorithm must be used in order to find the reconstruction with maximal membership in the intersection set. This is a nonlinear, large scale optimization problem and the use of special purpose computer architectures is unavoidable for the solution in a short time.

\section{Results and discussions}

The simulation result in fig. 2 demonstrates the improvement obtained by the new method over the conventional filtered backprojection. In the simulation, 32 views with 64 detectors are used to reconstruct the Shepp-Logan phantom on a 64 X64 grid. To obtain the improved result, the nonnegativity of the reconstruction, approximate ranges for the CT numbers and region of support are assumed to be known a priori.

Clearly, the new method is capable of generating better reconstructions. Its main problem is the computational complexity. Using the existing technology, the new method can be used in those cases where it is vital to have a reconstruction consistent with all 
available information. However, with the improvements in computing hardware, it will soon be possible to use more complicated methods in order to get closer to reality.

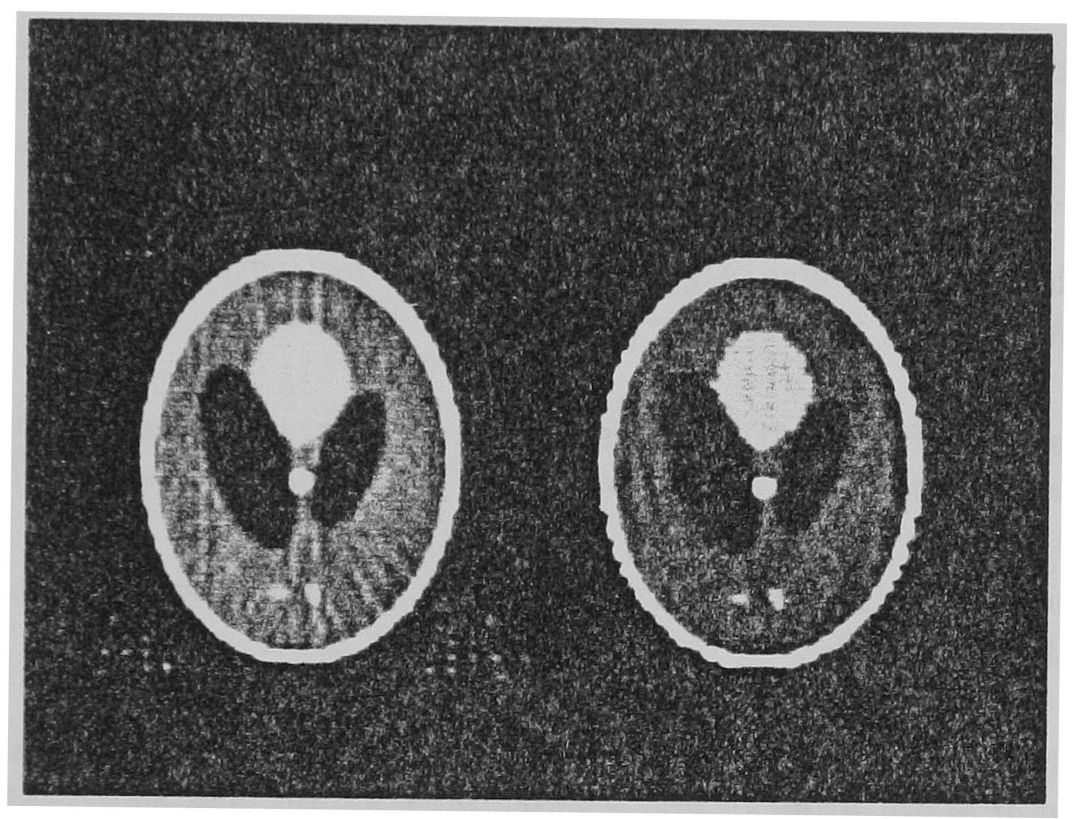

Figure 2 - (Left) Filtered backprojection, (right) new method.

\section{References}

[1] K.C.Tam, V.Perez-Mendez, "Tomographical Imaging with Limited Angle Input," J. Opt. Soc. Am., vol. 71, pp. 582-592, May 1981.

[2] B.P.Medoff, W.R.Brody, M.Nassi, A. Macovski, "Iterative Convolution Backprojection Algorithms for Image Reconstruction from Limited Data," J. Opt. Soc. Am., vol. 73, pp. 1493-1500, Nov. 1983.

[3] M.I.Sezan, H.Stark, "Tomographic Image Reconstruction from Incomplete View Data by Convex Projections and Direct Fourier Inversion," IEEE Trans. Med. Img., vol. MI-3, - n1 no Trno Figure 2 - (Left) Filtered backprojection, (right) new method. 
[4] D.Dubois, H.Prade, "Fuzzy Sets and Systems: Theory and Applications," Academic Press, New York, 1980.

[5] Y.Censor, "Finite Series Expansion Reconstruction Methods," Proc. IEEE, vol. 71, pp. 409-419, March 1983.

[6] M.H.Buonocore, W.R.Brody, A.Macovski, "A Natural Pixel Decomposition for Two Dimensional Image Reconstruction," IEEE Trans. Biomed. Eng., vol. BME-28, pp. 69-78, Feb. 1981.

[7] P.M.Joseph, "An Improved Algorithm for Reprojecting Rays Through Pixel Images," IEEE Trans. Med. Img., vol MI-1,'pp. 192-196, Nov. 1982.

[8] M.R. Civanlar, H.J.Trussell, "Consistency in Tomographic Reconstruction by Iterative Methods," Proceedings of the IEEE Int. Conf. on Acoust. Speech and Sig. Proc., Tokyo, Japan, April 1986.

[9] - "Constructing Membership Functions Using Statistical Data," Int. J. for Fuzzy Sets and Systems, January 1986.

[10] A. Lent, H.Tuy, "An Iterative Method for the Extrapolation of Band Limited Functions", Journal of Math. Analysis and Applications, 83, pp. 554-565, 1981.

[11] H.J. Trussell, M.R. Civanlar, "Feasible Solution in Signal Restoration", IEEE, Trans. Acoustic Speech and Signal Processing, Vol. ASSP-32, No.2, pp. 201-212, April 1984.

[12] L.M. Bregman, "The Method of Successive Projection for Finding a Common Point of Convex Sets", Soviet Math. Doklady, vol. 6, pp. 688-692, 1965.

[13] L.G. Gubin, B.T. Polyak, E.V. Raik, "The Method of Projections for Finding the Common Point of Convex Sets", USSR Computational Mathematics and Mathematical Physics, 7, no. 6, pp 1-24,1967. 